\title{
Undergrading and Understaging in Patients with Clinically Insignificant Prostate Cancer who Underwent Radical Prostatectomy
}

\author{
Irai S. Oliveira, Jose Pontes-Junior, Daniel K. Abe, Alexandre Crippa, Marcos F. Dall'Oglio, \\ Adriano J. Nesralah, Katia R. M. Leite, Sabrina T. Reis, Miguel Srougi
}

Urology Department, University of Sao Paulo Medical School, Sao Paulo, Brazil

\begin{abstract}
Purpose: The aim of our study is to evaluate the undergrading and understaging rates in patients with clinically localized insignificant prostate cancer who underwent radical prostatectomy.

Materials and Methods: Between July 2005 and July 2008, 406 patients underwent radical prostatectomy for clinical localized prostate cancer in our hospital. Based on preoperative data, 93 of these patients fulfilled our criteria of non-significance: Gleason score $<7$, stage T1c, PSA $<10 \mathrm{ng} / \mathrm{mL}$ and percentage of affected fragments less than $25 \%$. The pathologic stage and Gleason score were compared to preoperative data to evaluate the rate of understaging and undergrading. The biochemical recurrence free survival of these operated insignificant cancers were also evaluated.

Results: On surgical specimen analysis $74.7 \%$ of patients had Gleason score of 6 or less and $25.3 \%$ had Gleason 7 or greater. Furthermore $8.3 \%$ of cases showed extracapsular extension. After 36 months of follow-up 3.4\% had biochemical recurrence, defined by a PSA above $0.4 \mathrm{ng} / \mathrm{mL}$.

Conclusions: Despite the limited number of cases, we have found considerable rates of undergrading and understaging in patients with prostate cancer whose current definitions classified them as candidates for active surveillance. According to our results the current definition seems inadequate as up to a third of patients had higher grade or cancer outside the prostate.
\end{abstract}

Key words: prostate neoplasms; Gleason score; prostatectomy

Int Braz J Urol. 2010; 36: 292-9

\section{INTRODUCTION}

Prostate cancer (PC) is the most commonly diagnosed malignancy among males in western countries. Autopsy studies estimate that $30 \%$ of men over 50 years harbors histological PC $(1,2)$, but the chance of clinical diagnosis is much lower, being approximately $11 \%$ during lifetime (3), meaning that not all PC needs curative treatment. According to Epstein et al. (4), 16\% of nonpalpable PC diagnosed by screening techniques is insignificant and may be safely managed conservatively.
For this reason, active surveillance protocols have been proposed as an option for patients with both low grade and stage, and several studies to date have shown the feasibility of treating localized PC by expectant or conservative procedures with good results (5). These studies reported a dropout rate of 25 to $30 \%$ driven mainly by tumor progression or patient and physician anxiety.

The main factor determining success in active surveillance protocols is the proper selection of patients. Due to the large PC heterogeneity, it is of paramount importance to distinguish the patients with 
biologically aggressive tumors that need definitive treatment from those with an indolent tumor that benefits more by active surveillance $(5,6)$.

Currently, the patients are selected accordingly to specific findings at biopsy and clinical stage, but the criteria of clinical non-significance are variable. The most widely accepted is the Epstein criteria, which consist of prostatic specific antigen (PSA) density $0.1-0.15$, low or intermediate cancer grade, core involvement less than $3 \mathrm{~mm}$ and involvement of only one needle biopsy core (4). These criteria are used to predict the presence of clinically insignificant tumor, defined by Gleason patterns less than 4 , tumor volume less than $0.5 \mathrm{~cm}^{3}$ and organ-confined disease (4).

However, one should not forget the known existence of understaging and undergrading for any neoplasm which can erroneously engage patients in expectant management when local treatment was the best option.

The incorrect staging and grading is a real threat to any active surveillance protocol. A study evaluating surgical specimens of patients with PSA less than $10 \mathrm{ng} / \mathrm{mL}$, which is associated with lower stages, showed extra-capsular extension or seminal vesicle involvement in $10 \%$ and $3 \%$ of cases respectively (7).

Furthermore, Gleason score discordance between biopsy and surgical specimen has been estimated to vary between 47 to $69 \%(8,9)$. A metanalysis involving over 14,000 patients, found that the Gleason graduation of prostatectomy was correctly anticipated by the biopsy in $63 \%$ of the patients. Interestingly, among all patients with high-grade tumor in the surgical specimen, $67 \%$ had tumors of low or moderate grade in the biopsy, indicating a higher risk of undergrading for these patients (10).

In conclusion, these studies reflects the inaccuracy of current staging and grading regarding the true insignificance of $\mathrm{PC}$, meaning that selection of patients is of crucial importance in active surveillance protocols. The aim of this study was to compare data of prostate biopsy with the results of surgical specimen of patients with clinically insignificant operated $\mathrm{PC}$, in order to evaluate the rate of undergrading and understaging. We also evaluated the biochemical recurrence free survival for these patients.

\section{MATERIALS AND METHODS}

Between July 2005 and July 2008 a group of 406 men diagnosed with localized PC underwent radical prostatectomy at our institution. From this group, we selected the patients whose tumor was diagnosed by an extended biopsy protocol and who fulfilled the following criteria of non-significance: preoperative PSA less than or equal to $10 \mathrm{ng} / \mathrm{mL}$, staging clinical T1c, transrectal prostate biopsy with Gleason grading less than or equal to 6 , no pattern Gleason 4 or 5 and percentage of affected fragments less than or equal to $25 \%$ (Table-1). Patients who received hormone therapy before surgery were excluded. Considering these criteria, 93 patients were selected for this analysis.

The following surgical pathology data was recorded: Gleason score, pathological staging, seminal vesicle invasion, microvascular and perineural invasion, extracapsular invasion, bladder neck invasion, positive margin and total weight of prostate. Unfortunately, tumoral volume, an important predictor of biological behavior, is not a parameter routinely measured in our institution and was not recorded.

We compared the Gleason score concordance between biopsy and surgical specimen and the percentage of patients with locally advanced disease, attempting to estimate the number of patients erroneously classified as candidates to active surveillance. The biochemical recurrence free survival was calculated considering recurrence as a PSA above $0.4 \mathrm{ng} / \mathrm{mL}$.

\section{Statistical Analyses}

The chi-square test was employed to evaluate the difference of the Gleason score between biopsy

Table 1 - Definition of clinically insignificant prostate cancer.

\begin{tabular}{lc}
\hline PSA preoperative & $<10 \mathrm{ng} / \mathrm{mL}$ \\
Clinical stage & $\mathrm{T} 1 \mathrm{c}$ \\
Gleason score in biopsy & $\leq 6$ \\
$\%$ of positive cores in biopsy & $\leq 25 \%$ \\
\hline
\end{tabular}

PSA = protein-specific antigen. 
Table 2 - Demographic data.

\begin{tabular}{lc}
\hline Variable & N. of Patients \\
\hline Total & 93 \\
Age (mean) & 65.7 years (range $48-79)$ \\
PSA preoperative (mean) & $6.03 \mathrm{ng} / \mathrm{mL}(2.5-9.7)$ \\
N. of cores in biopsy (mean) & $12.01(10-18)$ \\
$\%$ of positive cores (mean) & $14.6 \%(5.6-25 \%)$ \\
\hline
\end{tabular}

PSA = prostatic specific antigen.

Table 3 - The comparison of Gleason scores between biopsy and surgical specimen in 87 cases.

\begin{tabular}{lllcccc}
\hline \multicolumn{7}{c}{ Surgical Specimen } \\
\hline Biopsy & 4 & 5 & 6 & 7 & 8 & Total \\
4 & 0 & 0 & 1 & 1 & 0 & 2 \\
5 & 0 & 0 & 2 & 2 & 0 & 4 \\
6 & 0 & 2 & 60 & 16 & 3 & 81 \\
Total & 0 & 2 & 63 & 19 & 3 & 87 \\
\hline
\end{tabular}

Chi-square $p<0.001$

and surgical specimen and a $\mathrm{p}$ value $<0.05$ was considered statically significant. For other variables the statistical methods consisted of descriptive and categorical analyses.

\section{RESULTS}

The average age of patients was 65.7 years and the mean PSA was $6.03 \mathrm{ng} / \mathrm{mL}$. The average percentage of positive fragments on biopsy was $14.6 \%$. The complete demographic data is depicted in Table- 2 .

The comparison of Gleason score between biopsy and surgical specimen was possible in 87 cases and the results are displayed in Table-3. According to this table, $77.9 \%$ of cases showed the same Gleason score, while upgrading and undergrading occurred in $19.5 \%$ and $2.6 \%$ of cases, respectively. Employing the Chi-square test, a significant difference $(p<0.001)$ of Gleason score between radical prostatectomy specimen and biopsy was observed, being important to note that $25 \%$ of clinically insignificant PC showed Gleason score higher than 6 at surgery.
Regarding the pathological stage, data was available for 84 patients of which $90.4 \%$ had organ confined disease (Table-4). Additional surgical pathology data is showed in Table-5.

After 36 months of follow-up only three patients $(3.4 \%)$ had biochemical recurrence defined as a PSA greater than or equal to $0.4 \mathrm{ng} / \mathrm{mL}$.

\section{COMMENTS}

The discrepancy between the Gleason score observed at the biopsy and surgical specimen may

Table 4 - Pathological stage (TNM) of patients.

\begin{tabular}{lc}
\hline Pathological Stage & N. of Patients \\
\hline T0 & $3(3.6 \%)$ \\
T2 & $73(86.9 \%)$ \\
T3 & $8(9.5 \%)$ \\
Total & $84(100 \%)$ \\
\hline
\end{tabular}

TNM = tumor, node, metastasis. 
Table 5 - Pathological characteristics of surgical specimens of radical prostatectomy.

\begin{tabular}{lcc}
\hline & Present & Absent \\
\hline Invasion of seminal vesicles & $6(7.1 \%)$ & $78(92.9 \%)$ \\
Extracapsular extension & $7(8.3 \%)$ & $77(92.0 \%)$ \\
Invasion of bladder neck & $1(1.2 \%)$ & $83(98.8 \%)$ \\
Positive margin & $6(7.1 \%)$ & $78(92.9 \%)$ \\
\hline
\end{tabular}

result in improper assessment of the disease and treatment, which can influence the prognosis of an individual patient, specially if active surveillance is proposed. Therefore, the correct stage and grade is of paramount importance in the treatment decision for any neoplasm.

In our series, we found a substantial Gleason score disagreement between biopsy and surgical specimen in patients that fulfilled active surveillance requirements $(\mathrm{p}<0.001)$. Within a group of patients with non palpable tumors of low grade, $25 \%$ had Gleason score of 7 or greater in the surgical specimen, reflecting the inadequacy of grade prediction with the current employed methods.

The undergrading rate of $25 \%$ underscores the risk and consequence of incorrect grade evaluation at biopsy in a group of patients that would be assigned to conservative management. In accordance to our results, Müntener et al. evaluating 6625 patients found an identical Gleason score in biopsy and surgical specimens only in a third of patients (8). In a contemporary series of 1,455 men who underwent radical prostatectomy at John Hopkins, although the rate of undergrading was smaller than before, the disagreement between biopsy and radical prostatectomy Gleason score was seen in $24 \%$ of cases (11), a rate similar to that observed in our study.

An important aspect of our results is that PC diagnosis was made through extended biopsy protocols, which is known to improve diagnosis and reduce the sampling error that is intrinsic to the ultrasoundguided prostate biopsy. The better performance of extended biopsy when compared to fewer samplings schemes can be exemplified by the Nesrallah's study, who found $\mathrm{PC}$ detection rates of $75 \%$ and $88 \%$ when 6 or 14 cores were respectively sampled (12). However, our undergrading rate was considered significant even when employing extended biopsy.

The precise staging is also important for adequate PC management. In our series of clinically insignificant patients, despite 3 cases that showed pT0, we found non organ confined disease in $9.5 \%$ of cases. This finding is a known negative prognostic factor in PC and does not qualify these tumors as being indolent.

A lower PSA is associated with organ confined tumor and is a common requisite of any clinically insignificant criteria, however there is sufficient data indicating that lower PSA is not always associated with indolent PC. A study evaluating surgical specimens of patients with PSA less than $4.0 \mathrm{ng} / \mathrm{mL}$ revealed extra-prostatic extension or positive margin in $8.3 \%$ of cases (13). Likewise, Geary et al. (7) found positive surgical margins in 13\% of non palpable tumors with PSA between 4 and 10 $\mathrm{ng} / \mathrm{mL}$.

It is noteworthy, that in our series the error related to staging $(9.5 \%)$ was lower than the error rate related to grade assignment (25\%), a finding that was also observed by others (14), which indicates that new methods should be particularly developed to improve grade prediction in PC.

Considering the undergrading and understaging together, we observed that up to a third of our patients with clinically insignificant tumors displayed unfavorable findings at radical prostatectomy. In agreement with our results, Chun et al. evaluating patients with clinically insignificant tumor found that $33 \%$ had pathological Gleason score of 7 to 10 or non organ confined tumor at surgical specimen (15). Even when the cohort was restricted to patients who also had PSA $<10$ and T1c clinical stage the rate of unfavorable cancer was $28 \%$ (15). 
Similarly, D'Amico et al. evaluated 66 men with PC diagnosed on the basis of a single microscopic examination of adenocarcinoma, and found extracapsular extension in $4 \%$ and positive margin in $6 \%$ of cases (16). It is important to mention that $10 \%$ of these patients failed biochemically within 5 years after radical prostatectomy.

Even after a short follow-up period, we observed that three patients (3.4\%) had biochemical recurrence. Likewise, a systematic review of operated small-volume cancer on biopsy showed biochemical recurrence in $8.6 \%$ of cases (range $6.1 \%-12.1 \%$ ) (17). These data emphasize the fact that even clinically insignificant cancer may not be cured by radical prostatectomy.

We believe that active surveillance is an adequate treatment for $\mathrm{PC}$, however a considerable proportion of patient candidates for this modality of treatment have "significant" features at surgical specimen. Our data indicates that current criteria to select patients for active surveillance seems inappropriate, as once up to a third of these patients clearly do not have insignificant tumors; in fact, they would be exposed to mortality by PC if the tumor was left untreated.

In accordance to our conclusion, a recent validation of Epstein criteria in European men showed that $24 \%$ of the patients who fulfilled the criteria had unfavorable characteristics at radical prostatectomy (14). The authors conclude that the widely used Epstein criteria underestimate the true nature of $\mathrm{PC}$ and that caution is advised when treatment decisions are based solely on this single criterion (14).

Corroborating this observation, Goto et al. evaluated 170 surgical specimens whose data fulfill the Ohori criteria of non-significance, which are PSA density less than 0.1 , clinical stage T1c and maximum length of cancer of $2 \mathrm{~mm}$ in any core, and found that $25 \%$ of specimens showed significant PC (18). These two series, along with ours, indicate that the undergrading and understaging rates are similar in clinically insignificant PC whatever the criteria employed.

It is important to note that the Epstein criteria were largely validated $(14,19)$ and, although not perfectly accurate, remain the better alternative for prediction of clinically insignificant PC when compared to other definitions $(18,20)$. The Epstein criteria are more accurate, for example, than the Kattan nomogram whose accuracy is between $64 \%$ and $79 \%(20)$.

The addition of molecular biology data may add to the predictive accuracy of the existing criteria for clinical non-significance, as demonstrate by Kattan et al. that increased the accuracy of biochemical recurrence prediction by adding TGF- $\beta$ e interleukin 6 levels in previous nomogram (21). The inclusion of PSA derivatives may also improve prediction and a study evaluating 163 radical prostatectomy specimens of stage T1c showed that the addition of free PSA increased the accuracy of Epstein criteria (22).

We recognize that our small patient population is a limitation to our conclusions, due to the fact that our institution is a tertiary health care center that receives the more complex and advanced cases. Therefore, only a few of our operated patients could be included in this analysis. Nevertheless, based in our results, other criteria should be developed in order to improve the non-significance factor and selection of PC patients, and to reduce the understaging and undergrading rates.

\section{CONCLUSION}

Although the expectant management for PC is a valid alternative treatment of properly selected cases, after analyzing our data we conclude that special care should be taken when including patients in this modality of treatment, because the risk of under staging and under grading seems substantial even in these properly selected cases.

\section{ACKNOWLEDGEMENTS}

Mrs. Rita Ortega helped in preparing this paper and Mrs. Adriana Sañudo performed the statistical analysis.

\section{CONFLICT OF INTEREST}

None declared. 


\section{REFERENCES}

1. Franks LM: Proceedings: Etiology, epidemiology, and pathology of prostatic cancer. Cancer. 1973; 32: 1092-5.

2. Hølund B: Latent prostatic cancer in a consecutive autopsy series. Scand J Urol Nephrol. 1980; 14: 2935.

3. Canadian Cancer Society/National Cancer Institute of Canada: Canadian Cancer Statistics 2000, Toronto, Canada, 2000.

4. Epstein JI, Walsh PC, Carmichael M, Brendler CB: Pathologic and clinical findings to predict tumor extent of nonpalpable (stage $\mathrm{T} 1 \mathrm{c}$ ) prostate cancer. JAMA. 1994; 271: 368-74.

5. Choo R, Klotz L, Danjoux C, Morton GC, DeBoer G, Szumacher E, et al.: Feasibility study: watchful waiting for localized low to intermediate grade prostate carcinoma with selective delayed intervention based on prostate specific antigen, histological and/or clinical progression. J Urol. 2002; 167: 1664-9.

6. Stamey TA, McNeal JE, Yemoto CM, Sigal BM, Johnstone IM: Biological determinants of cancer progression in men with prostate cancer. JAMA. 1999; 281: 1395-400.

7. Geary ES, Stamey TA: Pathological characteristics and prognosis of nonpalpable and palpable prostate cancers with a Hybritech prostate specific antigen of 4 to $10 \mathrm{ng} . / \mathrm{mL}$. J Urol. 1996; 156: 1056-8.

8. Müntener M, Epstein JI, Hernandez DJ, Gonzalgo ML, Mangold L, Humphreys E, et al.: Prognostic significance of Gleason score discrepancies between needle biopsy and radical prostatectomy. Eur Urol. 2008; 53: 767-75; discussion 775-6.

9. Guimaraes MS, Quintal MM, Meirelles LR, Magna LA, Ferreira U, Billis A: Gleason score as predictor of clinicopathologic findings and biochemical (PSA) progression following radical prostatectomy. Int Braz J Urol. 2008; 34: 23-9.

10. Cohen MS, Hanley RS, Kurteva T, Ruthazer R, Silverman ML, Sorcini A, et al.: Comparing the Gleason prostate biopsy and Gleason prostatectomy grading system: the Lahey Clinic Medical Center experience and an international meta-analysis. Eur Urol. 2008; 54: 371-81.

11. Fine SW, Epstein JI: A contemporary study correlating prostate needle biopsy and radical prostatectomy Gleason score. J Urol. 2008; 179: 1335-8; discussion 1338-9.

12. Nesrallah L, Nesrallah A, Antunes AA, Leite KR, Srougi M: The role of extended prostate biopsy on prostate cancer detection rate: a study performed on the bench. Int Braz J Urol. 2008; 34: 563-70; discussion 570-1.

13. Leite KR, Srougi M, Dall'Oglio MF, Sanudo A, Camara-Lopes LH: Histopathological findings in extended prostate biopsy with PSA $<$ or $=4 \mathrm{ng} / \mathrm{mL}$. Int Braz J Urol. 2008; 34: 283-90; discussion 290-2.

14. Jeldres C, Suardi N, Walz J, Hutterer GC, Ahyai S, Lattouf JB, et al.: Validation of the contemporary epstein criteria for insignificant prostate cancer in European men. Eur Urol. 2008; 54: 1306-13.

15. Chun FK, Suardi N, Capitanio U, Jeldres C, Ahyai S, Graefen M, et al.: Assessment of pathological prostate cancer characteristics in men with favorable biopsy features on predominantly sextant biopsy. Eur Urol. 2009; 55: 617-28-6.

16. D'Amico AV, Wu Y, Chen MH, Nash M, Renshaw AA, Richie JP: Pathologic findings and prostate specific antigen outcome after radical prostatectomy for patients diagnosed on the basis of a single microscopic focus of prostate carcinoma with a gleason score $</=$ 7. Cancer. 2000; 89: 1810-7.

17. Harnden P, Naylor B, Shelley MD, Clements H, Coles B, Mason MD: The clinical management of patients with a small volume of prostatic cancer on biopsy: what are the risks of progression? A systematic review and meta-analysis. Cancer. 2008; 112: 971-81. Erratum in: Cancer. 2008; 112: 2101.

18. Goto Y, Ohori M, Arakawa A, Kattan MW, Wheeler TM, Scardino PT: Distinguishing clinically important from unimportant prostate cancers before treatment: value of systematic biopsies. J Urol. 1996; 156: 105963.

19. Bastian PJ, Mangold LA, Epstein JI, Partin AW: Characteristics of insignificant clinical T1c prostate tumors. A contemporary analysis. Cancer. 2004; 101: 2001-5.

20. Kattan MW, Eastham JA, Wheeler TM, Maru N, Scardino PT, Erbersdobler A, et al.: Counseling men with prostate cancer: a nomogram for predicting the presence of small, moderately differentiated, confined tumors. J Urol. 2003; 170: 1792-7.

21. Kattan MW, Shariat SF, Andrews B, Zhu K, Canto E, Matsumoto K, et al.: The addition of interleukin-6 soluble receptor and transforming growth factor beta 1 improves a preoperative nomogram for predicting biochemical progression in patients with clinically localized prostate cancer. J Clin Oncol. 2003; 21: 3573-9. 
22. Epstein JI, Chan DW, Sokoll LJ, Walsh PC, Cox JL, Rittenhouse H, et al.: Nonpalpable stage T1c prostate cancer: prediction of insignificant disease using free/ total prostate specific antigen levels and needle biopsy findings. J Urol. 1998; 160: 2407-11.
Accepted after revision:

November 3, 2009

\section{Correspondence address:}

José Pontes Jr.

Av. Dr. Arnaldo, 455 / 2nd floor LIM 55

Sao Paulo, SP, 01246-903, Brazil

Fax: + $55113061-7183$

E-mail: docjpjr@uol.com.br

\section{EDITORIAL COMMENT}

In this study, Santana de Oliveira et al. (1) report on how current criteria for clinically insignificant prostate cancer $(\mathrm{PCa})$ work in their series which includes less than 100 cases. This is an important limitation to the study design; likewise, the paper is of interest since insignificant $\mathrm{PCa}$ is an important topic in daily practice. As shown by Santana de Oliveira et al. (1) we do not have a reliable model to predict insignificant prostate cancer in every single patient. Prediction of clinically insignificant prostate cancer (PCa) remains as a major problem in clinical practice. In the updated format, the contemporary Epstein criteria represent the most widely used tool for prediction of clinically insignificant prostate cancer, in spite of limitations. Of 217 patients with organ confined disease, $18(7.6 \%)$ had Gleason sum 7 or higher in the series used to update the Epstein criteria. Therefore, 199 of 237 patients $(83.9 \%)$ in the updated Epstein criteria series had both organ-confined disease and favorable (Gleason 6 or lower) prostate cancer grade. This finding indicates that the updated Epstein criteria underestimated disease stage and/or grade in 16\% of North American patients and were accurate in $84 \%$ of predictions. Conversely, the rate of Gleason sum 7 was substantially higher in Brazilian population (25.3\%) which yielded substantially lower overall accuracy $(74.7 \%)$ than the one reported in North America (84\%); the Brazilian cohort refers to 12 (1018) cores per case just similar to the Hopkins study that refers to 12 core biopsies. Therefore, it may be argued that the stage and grade migration that results in the detection of an increasing proportion of Gleason 6 prostate cancer may result in lower error rate of the Epstein clinically insignificant prostate cancer criteria, when these are compared with Brazilian findings (1-2). The authors (1) provide an in depth review of the various causes leading to failure of the contemporary Epstein's criteria. An important issue derived from the current study deserves a comment since it is related to the diagnostic rate of Gleason 7; this grade is heterogeneous and represents the most complex exercise in needle prostate biopsies sign out, and differences in the performance of the Epstein criteria between North America and Brazil may explain by itself the observed differences seen in the current study. The results by Santana de Oliveira et al. (1) 
emphasize the need for continuing education activities concerning Gleason grading in order to achieve the maximum accuracy and reproducibility in daily practice of prostate pathology.

\section{REFERENCES}

1. Oliveira IS, Pontes-Junior J, Abe DK, Crippa A, Dall'oglio M, Nesrallah AJ, et al.: Undergrading and understaging in patients with clinically insignificant prostate cancer who underwent radical prostatectomy. Int Braz J Urol. 2010, In Press.

2. Montironi R, Mazzucchelli R, Scarpelli M, LopezBeltran A, Mikuz G: Prostate carcinoma I: prognostic factors in radical prostatectomy specimens and pelvic lymph nodes. BJU Int. 2006; 97: 485-91.

Dr. Antonio Lopez Beltran Department of Pathology, School of Medicine University of Cordoba Cordoba, Spain E-mail:em1lobea@uco.es

Dr. Rodolfo Montironi Institute of Pathological Anatomy Polytechnic University of the Marche Region Ancona, Italy

Dr. Liang Cheng Department of Pathology and Laboratory Medicine Indiana University School of Medicine Indianapolis, IN, USA 